\title{
Coherent X-ray Imaging of CO-Adsorption-Induced Structural Changes in Pt Nanoparticles: Implications for Catalysis
}

\author{
Manuel Abuin, ${ }^{\dagger}$ Young Yong Kim, ${ }^{\dagger}{ }^{\circ}$ Henning Runge, ${ }^{\dagger \dagger}{ }^{\dagger}$ Satishkumar Kulkarni, ${ }^{\dagger}$ Simon Maier, ${ }^{\S}$ \\ Dmitry Dzhigaev, ${ }^{\| \odot}$ Sergey Lazarev, ${ }^{\dagger, \perp}$ Luca Gelisio, ${ }^{\dagger}$ Christoph Seitz, ${ }^{\dagger}$ CMarie-Ingrid Richard,, \&,

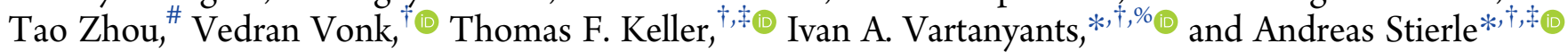 \\ ${ }^{\dagger}$ Deutsches Elektronen-Synchrotron (DESY), D-22607 Hamburg, Germany \\ ${ }^{\ddagger}$ Physics Department, University of Hamburg, D-20355 Hamburg, Germany \\ ${ }^{\S}$ University of Regensburg, D-93053 Regensburg, Germany \\ "Division of Synchrotron Radiation Research, Department of Physics, Lund University, SE-221 00 Lund, Sweden \\ ${ }^{\perp}$ National Research Tomsk Polytechnic University (TPU), 634050 Tomsk, Russia \\ \# European Synchrotron Radiation Facility (ESRF), F-38043 Cedex Grenoble, France \\ \&Aix-Marseille Université, CNRS, Université de Toulon, IM2NP UMR 7334, 13397 Marseille, France \\ ${ }^{\%}$ National Research Nuclear University MEPhI, 115409 Moscow, Russia
}

\section{Supporting Information}

\begin{abstract}
Using coherent X-ray diffraction imaging (CXDI) as an in situ tool, we determined the shape and strain state of a platinum nanoparticle with $\approx 160 \mathrm{~nm}$ diameter supported by a strontium titanate substrate. The experiment was performed at a temperature of $400 \mathrm{~K}$ under continuous gas flow conditions of pure $\mathrm{Ar}$ and $\mathrm{Ar} / \mathrm{CO}$ mixtures. The nanoparticle was preselected by scanning electron microscopy (SEM) and postanalyzed by atomic force microscopy (AFM). We obtain a very good agreement between the overall nanoparticle size, shape, and defect structure as determined by CXDI and AFM. In addition, we compare the strain state in the nanoparticle near surface region and its bulk: For pure Ar

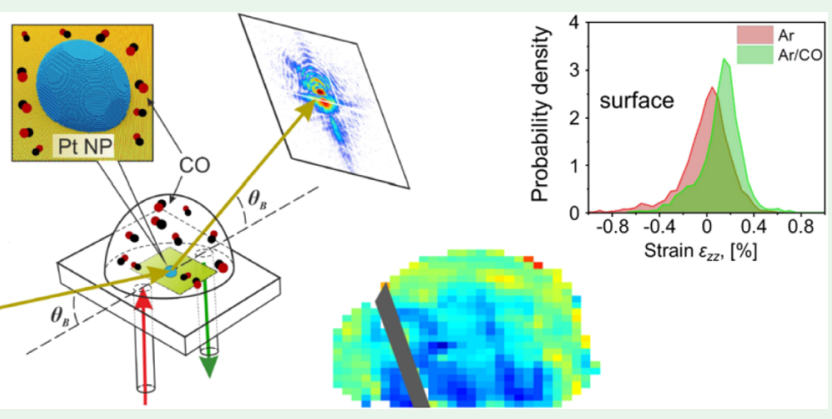
flow, we find a slight compressive strain in the nanoparticle bulk compared to an expansion in the near surface region. We ascribe the latter to the presence of high index vicinal surfaces. Our analysis suggests that under mixed Ar/CO flow at $400 \mathrm{~K}$ reshaping of the nanoparticle occurred. New high index facets developed, leading to a stronger lattice expansion, also propagating into the nanoparticle bulk. Our high-resolution experiments pave the way for future CXDI experiments under operando catalytic reaction conditions.
\end{abstract}

KEYWORDS: catalysis, single nanoparticle, in situ conditions, coherent diffraction imaging, hierarchical marker strategies

\section{INTRODUCTION}

Oxide-supported nanoparticles (NPs) play an eminent role as heterogeneous catalysts in exhaust gas cleaning, energy conversion, and storage. ${ }^{1}$ A detailed understanding of catalytic reactions is mandatory for the future development of heterogeneous catalysts with higher activity, selectivity, and improved lifetime. It requires an in-depth structural characterization of heterogeneous catalysts, representing a material with a rather complex morphology, as they are composed of oxidesupported metal nanoparticles. The metal nanoparticle shape is directly related to the catalytic behavior since it defines the possible adsorption sites, characterized by different local geometry, and therefore the absorption energy. ${ }^{2-8}$ Another important quantity is the nanoparticle's internal strain field, since it alters the adsorption probability and, thus, the activity and selectivity of the adsorption sites. ${ }^{9-14}$ Because only a fraction of strained surface atoms can alter the catalytic activity, the atomic site-specific strain determination plays an important role. $^{15}$

Conventional studies provide an averaged information over NP ensembles characterized by an intrinsic size, shape, strain, and orientational distribution resulting from the synthesis process, which makes the interpretation of the results often challenging. Heterogeneous catalytic reactions often imply atmospheric pressures and elevated temperatures, representing an additional obstacle for atomic scale characterization. ${ }^{16}$ One

Received: April 24, 2019

Accepted: July 26, 2019

Published: July 26, 2019 
possible way to overcome this so-called materials gap ${ }^{17}$ is the investigation of individual nanoparticles, which was so far the domain of transmission electron microscopy (TEM) only. ${ }^{18-20}$ However, electron microscopy suffers from a low penetration depth, which limits the size of the nanoparticles that can be studied. Moreover, environmental TEM exhibits a much lower flexibility with respect to realistic conditions at atmospheric pressures, gas mixtures, and elevated temperatures. ${ }^{21}$

A powerful method for the investigation of the structure of individual nanoparticles is coherent X-ray diffractive imaging (CXDI), ${ }^{22-24}$ which is intrinsically compatible with realistic reaction conditions. ${ }^{25-27}$ Bragg CXDI allows to retrieve the real space electron density distribution (i.e., the shape of a coherently scattering domain) as well as the strain distribution in the nanoparticle in three-dimensions (3D) with a spatial resolution better than $10 \mathrm{~nm} .{ }^{28-31}$ Here we report in situ Bragg CXDI experiments on a single Pt NP epitaxially grown on a $\mathrm{SrTiO}_{3}(001)$ substrate (STO) with a diameter of $160 \mathrm{~nm}$ and a height of $90 \mathrm{~nm}$, mimicking the typical situation in a heterogeneous catalyst. We studied the Pt nanoparticle under an $\mathrm{Ar} / \mathrm{CO}$ atmosphere at near atmospheric pressure flow conditions and at an elevated sample temperature of $400 \mathrm{~K}$. In contrast to previous reports on Bragg CXDI studies on single nano-objects, ${ }^{22-24,28-30}$ we preselected the nanoparticle prior to the X-ray experiment by scanning electron microscopy (SEM). The deposition of Pt-based markers inside the SEM allowed us to correlate our Bragg CXDI results with a detailed ex situ real-space analysis performed by SEM and atomic force microscopy (AFM). We observed that the Pt NP shape is terminated by vicinal surface facets producing long-ranged strain fields, which are altered in a characteristic way upon CO adsorption. Defects found in the Bragg CXDI reconstruction of the particle shape can be directly correlated to surface topographic features observed by AFM. Our results pave the way for future operando experiments at elevated pressures and temperatures on controlled, preselected single NPs using coherent X-ray imaging techniques and open up the possibility to address the role of local strain on the catalytic activity.

\section{EXPERIMENTAL SECTION}

For the nanofocus Bragg CXDI experiment, a sample with low NP density was prepared by physical vapor deposition of $\mathrm{Pt}$ at $1073 \mathrm{~K}$ onto a $\mathrm{TiO}_{2}$-terminated $\mathrm{STO}(001)$ single crystal surface under ultrahigh-vacuum conditions (see section 1 of the Supporting Information for more details). Afterward, the sample was annealed in a tube furnace in air for $600 \mathrm{~s}$ at $1373 \mathrm{~K}$ to induce the formation of NPs with a size in the range of $100 \mathrm{~nm}$, suitable for CXDI experiments. ${ }^{32}$ The ensemble of Pt NPs grew epitaxially with [001], [110], and [111] orientation along the substrate surface normal, as shown by grazing incidence X-ray diffraction (see Figures S1 and S2 and the discussion in section 2 of the Supporting Information). The particle under investigation is [111] oriented perpendicular to the substrate surface, and its in-plane reciprocal lattice $[11 \overline{2}]$ orientation is parallel to the STO substrate in-plane [100] direction.

The Bragg CXDI experiment was performed at the European Synchrotron Radiation Facility (ESRF), beamline ID01, at a photon energy of $8.5 \mathrm{keV}$. A sketch of the experimental setup is presented in Figure 1a. The X-ray beam was focused by a Fresnel zone plate to $300 \mathrm{~nm}$ horizontal and $150 \mathrm{~nm}$ vertical size (full width at halfmaximum). This resulted in a footprint of $520 \mathrm{~nm}$ along the beam for the employed incident angle of $18.75^{\circ}$. The diffraction patterns were recorded by a $2 \mathrm{D}$ MAXIPIX detector with $55 \mu \mathrm{m}$ pixel size installed at a sample-to-detector distance of $0.68 \mathrm{~m}$. The sample was mounted in a small flow cell equipped with a Be dome and a sample heater. ${ }^{33}$ Pure $\mathrm{Ar}$ and $\mathrm{Ar} / \mathrm{CO}$ mixtures were introduced by a computer controlled (a)
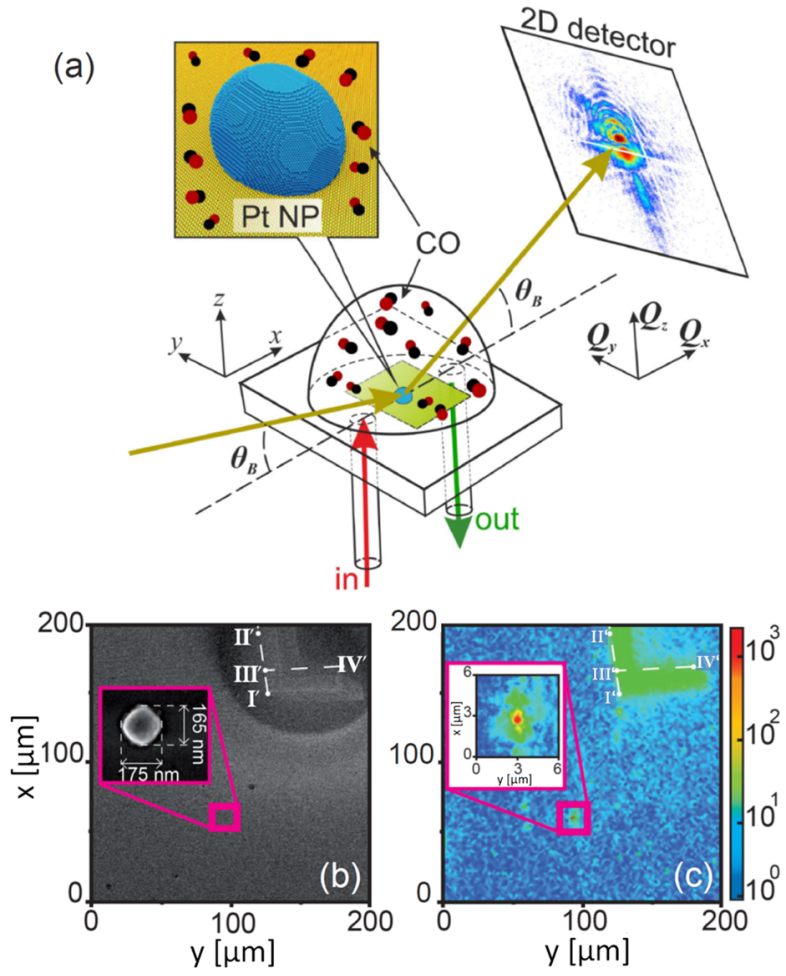

Figure 1. (a) Sketch of the experimental setup: the X-ray beam is focused by a Fresnel zone plate onto a single Pt NP. Bragg coherent diffraction patterns are collected by a $2 \mathrm{D}$ detector while the sample is exposed to a computer-controlled gas flow. (b) SEM image and (c) Pt 111 Bragg diffraction map obtained by a continuous fast-scan mode of the areas of interest. The L-shaped Pt-based marker with four reference points (I, II, III, IV) is used to relocate the preselected Pt NP inside the pink square. A zoom around the Pt NP is shown in both cases, see also section 4 of the Supporting Information.

gas cabinet at a constant total flow of $20 \mathrm{~mL} / \mathrm{min}$ and a total pressure of $50 \mathrm{mbar}$ (see section 3 of the Supporting Information).

The typical time range for a CXDI scan was around $1 \mathrm{~h}$, and the catalytic conditions were changed typically after a few hours, taking the stabilization and realignment into account.

Platinum-based markers were prepared by ion beam and electron beam induced deposition (IBID and EBID) using a Pt-containing precursor gas in a hierarchical arrangement at the DESY NanoLab ${ }^{34}$ to retrieve the position of preselected NPs during the X-ray diffraction experiment (see Figure $1 \mathrm{~b}$ and Figures S3-S5). Postexperimental ex situ AFM topographic images of the Pt NP were recorded in tapping mode in air by using an oxide-sharpened silicon probe.

To relocate the region of interest previously identified in the SEM measurements, the sample was prealigned using the coarse Pt-based markers visible in the optical microscope mounted on the beamline diffractometer. After mounting the Be dome and stabilizing a temperature of $400 \mathrm{~K}$ in a continuous flow of $\mathrm{Ar}$ gas, the fine alignment was performed using the Pt 111 Bragg peak signal of the epitaxial NPs (see Figure S3b) and the Pt 111 powder ring of the nanocrystalline Pt-based markers (see Figure S3a). Both could be separated on the 2D detector, as shown in Figure S3. We employed a fast scan mode, ${ }^{35}$ during which the regions of interest of the $2 \mathrm{D}$ detector are continuously read while two orthogonal piezo stages raster the sample surface. The result of the map scan is given in Figure 1c, which allowed us to relocalize the NP of interest with respect to the fine "L"-shaped Pt-based marker (see section 4 of the Supporting Information). The small footprint of the X-ray beam assures that the diffraction pattern from a single nanoparticle is observed. 


\section{RESULTS AND DISCUSSION}

We collected sets of coherent Pt 111 Bragg diffraction data at $400 \mathrm{~K}$ under continuous flow of $20 \mathrm{~mL} / \mathrm{min}$ pure $\mathrm{Ar}$ and mixtures of $4 \mathrm{~mL} / \mathrm{min} \mathrm{CO}$ with $16 \mathrm{~mL} / \mathrm{min}$ Ar, respectively. Incident angles $\theta$ of $\pm 0.6^{\circ}$ around the Pt 111 Bragg peak position were employed to map the $3 \mathrm{D}$ reciprocal space intensity distribution. Typical diffraction data at the $\mathrm{Pt} 111$ Bragg angle $\left(\theta=18.75^{\circ}\right)$ are shown in Figure S7. The 3D reciprocal space intensity distribution was obtained by interpolating diffraction patterns on a regular reciprocal space $Q_{x}, Q_{y}, Q_{z}$ grid (see Figures $S 8$ and $S 9$ and Movie $S 1$ and Movie S2 for more details). Slices of the intensity distribution in the $Q_{y}, Q_{z}$ plane in Figures 2a,b clearly show

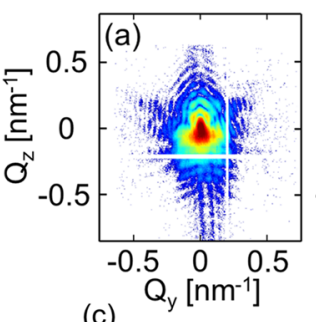

(c)

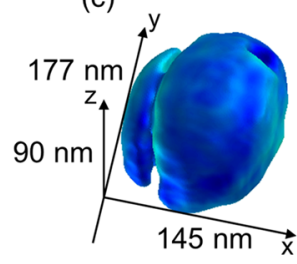

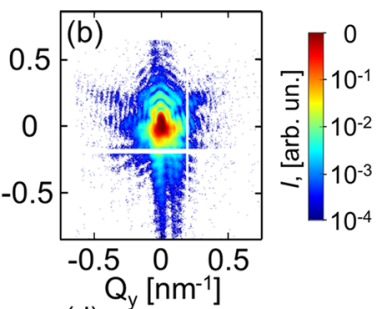

(d)

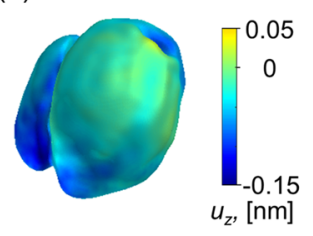

Figure 2. $Q_{y}, Q_{z}$ slices of the 3D intensity distribution shown in logscale of the Pt 111 Bragg reflection recorded at $400 \mathrm{~K}$ (a) under pure Ar flow and (b) under mixed Ar/CO flow. (c, d) Reconstructed NP shape and superimposed displacement field $u_{z}(r)$ as a color code for pure Ar flow and mixed Ar/CO flow, respectively.

interference arising from single particle coherent diffraction. Slight changes in the reciprocal space intensity distribution reflect the modification of the shape and strain field of the catalyst particle under different gas atmosphere conditions.

The real-space 3D structure of the NP, containing information about the shape and strain field, was reconstructed by an iterative phase retrieval approach ${ }^{31,36}$ as described in section 5 of the Supporting Information (see also Figures S10, S11, and S14, showing the reconstructed amplitude isosurface, the cross-section views of the reconstructed nanoparticle, the phase retrieval transfer function (PRTF) and the estimated resolution under pure $\mathrm{Ar}$ and $\mathrm{Ar} / \mathrm{CO}$ atmospheres). The largest size of the reconstructed voxels was achieved for Ar with a volume of $5.3 \times 4.2 \times 3.7 \mathrm{~nm}^{3}$ in $x$-, $y$-, and $z$-directions, respectively. The reconstruction results of the NP shape and displacement field $u_{z}(r)$ are shown in Figures $2 \mathrm{c}$,d. Because of the geometry of our experiment with the reciprocal space vector $\boldsymbol{H}$ directed perpendicular to the substrate, we are sensitive to the $z$-component of the displacement field $u_{z}(\boldsymbol{r}){ }^{37}$ The dimensions of the NP obtained from the CXDI reconstruction vary only marginally for different reaction conditions and are on average 145, 177, and $90 \mathrm{~nm}$ along the $x$-, $y$-, and $z$-directions, respectively. Because for the CXDI experiment we used hard X-rays that elastically scatter from deeply bound atomic levels, we are not sensitive to electronic structure modifications as, for example, charge transfer from adsorbates to the nanoparticle or reversely. The local displacement field $u_{z}(r)$ along the $\mathrm{Pt} 111$ reciprocal lattice vector was found to vary from -0.15 to $+0.05 \mathrm{~nm}$ over the whole NP. Upon exposure to mixed Ar/CO flow, the displacement field gets more positive at the NP surface, which will be discussed in more detail below.

To resolve morphological details of the $\mathrm{Pt} \mathrm{NP}$ and to confirm the reliability of the Bragg CXDI reconstruction, AFM topography measurements were performed. For the post-X-ray measurement SEM and AFM analysis, we switched to reducing conditions and then cooled the sample down to room temperature. Whereas from SEM analysis, the lateral size can be determined with nanometer precision, AFM can provide the NP height with nanometer resolution. In contrast, the size of a NP reconstructed by Bragg CXDI is a measure of the crystalline region that is contributing to the Bragg peak. A potentially smaller apparent particle height can arise, e.g., from interfacial dislocations originating from epitaxial strain to the STO substrate. Subsequent to the in situ X-ray Bragg CXDI experiment, the NP was relocated for postanalysis using AFM relying on the Pt-based markers. We achieved an unambiguous identification of the NP from the surrounding area as well as the shape and size of the NP itself (see Figures S5 and S6). The magnification in the inset of Figure $1 \mathrm{~b}$ indicates a mean lateral NP diameter of 165-175 nm. In Figure 3, we show the one-to-one correspondence of the CXDI reconstruction data and the post-AFM topography profile of the NP.

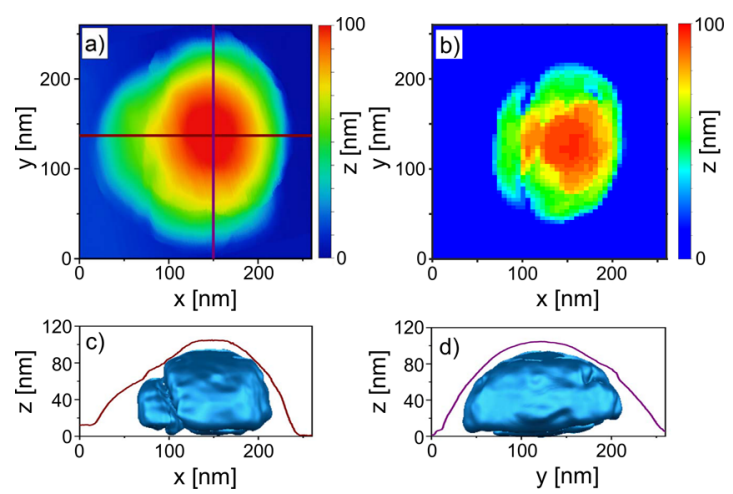

Figure 3. Comparison of (a) the AFM topography and (b) CXDI reconstruction of the single Pt NP in the $x-y$ plane. (c, d) To-scale overlay of the AFM profiles in the $x-z$ and the $y-z$ projection (violet lines) and the corresponding CXDI reconstructions of the NP oriented perpendicular to the shorter and longer diameter of the NP, respectively.

A slight asymmetry of the NP can be noted from the 2D AFM topography shown in Figure 3a. Indeed, the top view $(x-y$ plane) of the CXDI reconstructed NP in Figure $3 \mathrm{~b}$ confirms this NP asymmetry at a very similar lateral position and as such excludes an artifact in either of the two techniques. At this position, the reconstructed electron density distribution is separated into two parts by a region characterized by a lower amplitude, shown as a gap in Figure $3 \mathrm{~b}$. This region originates from a part of the sample not contributing to the 111 Bragg reflection measured for the CXDI reconstruction. Such a defect may arise from a twinned region of the NP, in which the "ABC" stacking of a set of $\{111\}$ type planes is reverted to "CBA" ${ }^{38,39}$ This hypothesis is supported by the inclination angle of the defect direction inside the bulk NP, which is in a good agreement with the Pt $11 \overline{1}$ plane orientation. Moreover, this defect obviously protrudes as a small kink out of the top 
surface of the NP, which is apparent in the AFM topography line profiles (see Figures 3c,d).

The close correspondence between the reconstructed Bragg CXDI and the AFM topography data of the NP is most obvious from Figures 3c,d. They contain different views of the 3D CXDI reconstruction of the NP along with an overlay of the corresponding AFM topographic line profile extracted from Figure $3 \mathrm{a}$ for both, the $x$ - and $y$-direction, respectively. Along the $y$-axis, the dimensions obtained by Bragg CXDI, SEM, and AFM are 177, 165, and $230 \mathrm{~nm}$ and, therefore, agree well when considering the lateral broadening arising from the convolution of the NP and the tip of the AFM. Along the $x$-axis, the Bragg CXDI dimension of the NP of $145 \mathrm{~nm}$ is shorter as compared to $175 \mathrm{~nm}$ obtained by SEM and $250 \mathrm{~nm}$ by AFM. We assign the reduced Bragg CXDI NP size to a missing part in the electron density reconstruction on the left side of the particle, likely to additional twin formation. Along the $z$-axis, the Bragg CXDI and AFM dimensions are 90 and $100 \mathrm{~nm}$, respectively, being again in close agreement. Therefore, we conclude that the AFM and SEM postanalysis validates the Bragg CXDI reconstruction and allows for a one-to-one correlation between the different methods.

Next, we will discuss the variation of the strain field in reaction to the mixed $\mathrm{Ar} / \mathrm{CO}$ flow exposure. Because of the geometry of our experiment, we are sensitive to the $z$ component $\varepsilon_{z z}(r)$ of the strain tensor $\varepsilon_{i j}(r)$ that is defined as

$$
\varepsilon_{z z}(\boldsymbol{r})=\frac{\partial u_{z}(\boldsymbol{r})}{\partial z}
$$

We point out that this strain tensor component depends on a position $r$ inside the particle. The color code in Figures $4 \mathrm{a}, \mathrm{b}$ indicates the local distribution of the $\varepsilon_{z z}$ component of the 3D strain field on the contour of the Bragg CXDI reconstructed $\mathrm{Pt}$ NP surface under Ar and Ar/CO flow, respectively.

The color distribution indicates that $\varepsilon_{z z}$ is locally varying on the NP surface and changes when switching from an Ar to

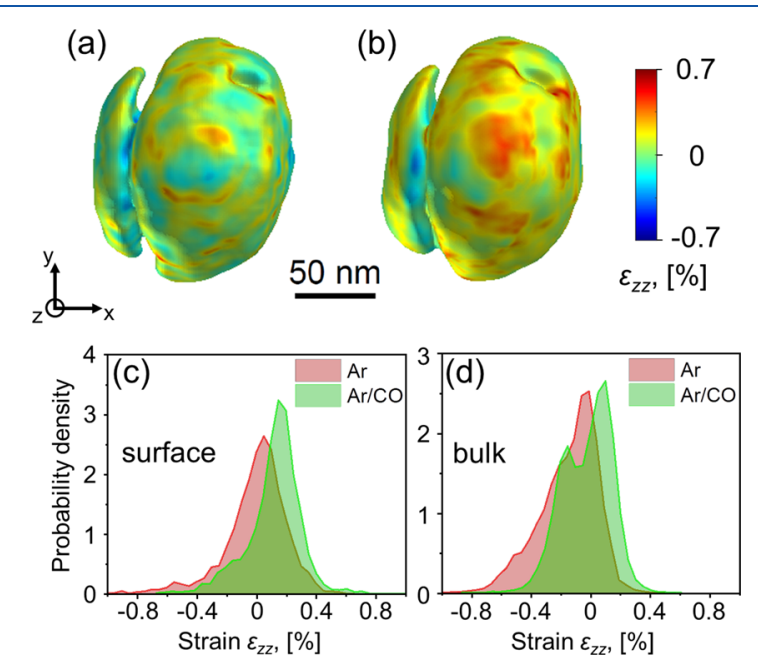

Figure 4. CXDI reconstruction results of the Pt NP under different conditions. Visualization of the $3 \mathrm{D}$ strain field component $\varepsilon_{z z}$ at a contour level of 0.1 (a) under Ar flow and (b) under mixed $\mathrm{Ar} / \mathrm{CO}$ flow; see also Movie S3 and Movie S4. (c) Probability density of the strain field in the NP near surface region and (d) in the bulk. The average near surface strain field changes from $-0.01 \%$ to $+0.12 \%$, and in the bulk it changes from $-0.15 \%$ to $-0.02 \%$, respectively, when switching from pure $\mathrm{Ar}$ to $\mathrm{Ar} / \mathrm{CO}$ flow.
$\mathrm{Ar} / \mathrm{CO}$ atmosphere. Locally, the absolute strain values on the $\mathrm{NP}$ surface are higher under $\mathrm{Ar} / \mathrm{CO}$ than under an $\mathrm{Ar}$ atmosphere, ranging from about $-0.7 \%$ of compressive strain to about $+0.7 \%$ of tensile $\varepsilon_{z z}$ strain values (see Figures $4 \mathrm{a}, \mathrm{b}$ ). In particular, we note most pronounced variations of the strain field on curved surface areas as compared to flat $\mathrm{Pt}$ facet terraces.

To quantitatively characterize the mean strain fields under inert $\mathrm{Ar}$ and $\mathrm{Ar} / \mathrm{CO}$ atmosphere, we determined histograms of the corresponding $\varepsilon_{z z}$ strain field components. The resulting histograms are shown in Figures $4 \mathrm{c}$, d for the surface and the bulk of the NP, for each of the two investigated gas environments. The histograms were obtained by a calculation of the strain in the bulk of the NP excluding the surface area and of the strain in the outermost surface layer of the NP (more details are discussed along with the three-dimensional representation of the strain distribution shown in Figure S15). In the NP near surface region an average strain field of $-0.01 \%$ was present under pure Ar flow. It gets shifted to a more positive value of $+0.12 \%$ under mixed $\mathrm{Ar} / \mathrm{CO}$ flow, which suggests that the $\mathrm{CO}$ adsorption induces an outward relaxation of the atoms at the near surface region. The average value of the strain field in the NP bulk under Ar atmosphere is $-0.15 \%$, while its value is $-0.02 \%$ under an $\mathrm{Ar} / \mathrm{CO}$ atmosphere. We also evaluated the standard deviation of the strain which gives an estimate of the uncertainty of our measurements (see Figure S13). This is in line with an overall shift of the compressive strain field in the bulk of the NP to more positive values, suggesting that the $\mathrm{CO}$ adsorption on the surface induces also an average lattice expansion in the core of the NP. This observation highlights the interplay between surface and bulk elastic response under gas adsorption conditions. Finite element modeling of a $200 \mathrm{~nm}$ sized Pt nanoparticle showed that the strain state at the edges and corners can also affect the strain state of the nanoparticle bulk. ${ }^{40}$ Because of its finite size, changes in the strain state by an adsorption-induced surface stress can be easier accommodated in the nanoparticle bulk as compared to bulk single crystals and may eventually be determined by CXDI being very sensitive to changes in the local strain state. It is worth mentioning that at $400 \mathrm{~K}$ and a $\mathrm{CO}$ partial pressure of $12.5 \mathrm{mbar}$ the $\mathrm{Pt} \mathrm{NP}$ surface is covered with a high-density CO phase. ${ }^{41}$ Because $\mathrm{CO}$ is not observed to dissociate on Pt nanoparticle surfaces, we rule out $\mathrm{C}$ and/or $\mathrm{O}$ diffusion into the bulk.

To rationalize our observations, it is instrumental to have a closer look at the NP shape and strain field distribution under pure $\mathrm{Ar}$ and $\mathrm{Ar} / \mathrm{CO}$ mixed flows in Figures 5a,b. Here, 2D strain field slices through the center of the NP in the (111)/ $(11 \overline{2})$ plane are plotted for pure Ar and Ar/CO flows. In both cases, the NP is terminated at the top and at the interface by low index (111) planes. In addition, higher index terminations by vicinal planes can be discerned, which can be directly assigned from a comparison with reciprocal space directions (see Figure S12a). Vicinal surfaces are characterized by a characteristic periodic arrangement of step and terrace sites, as pictured in the insets of Figure 5. They represent the NP surface termination well, since the NP shape reconstruction exhibits distinct facets with lateral extension much larger than the reconstruction voxel size. The vicinal (113), (551), and $(77 \overline{9})$ surfaces identified under Ar flow exhibit a typical terrace length of $1-2 \mathrm{~nm}$ (see the insets in Figures 5a,b). Atoms at the step edges of the vicinal surfaces exhibit strong inward relaxations and atoms on the bottom side of the step edges 


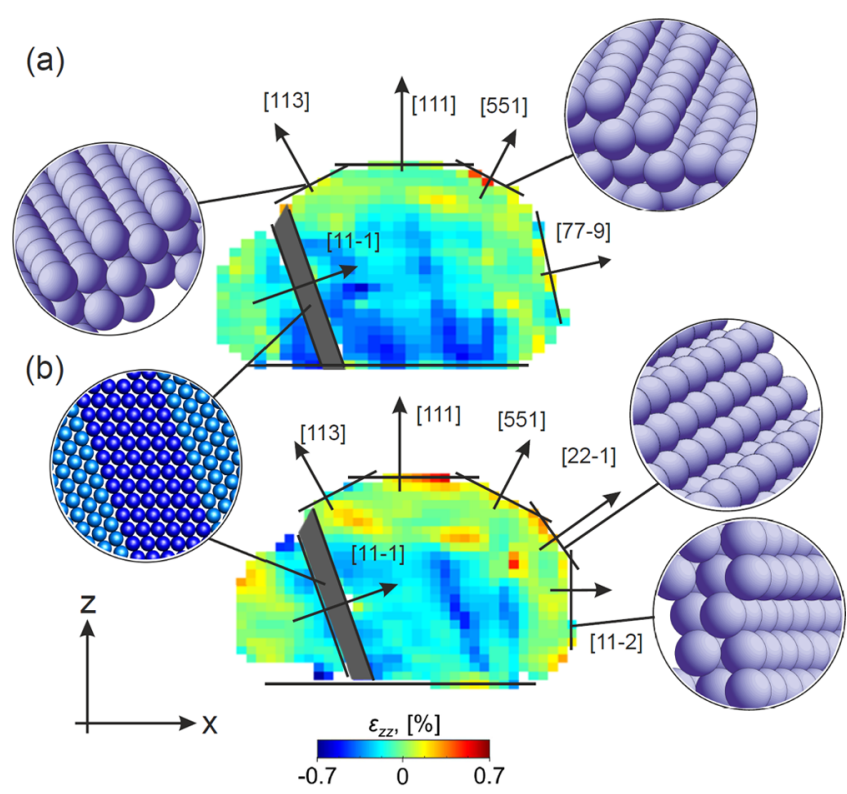

Figure 5. 2D slices of the $\epsilon_{z z}$ component of the strain field through the center of the reconstructed NP with a tentative assignment of the vicinal surface facets (a) under Ar flow and (b) under $\mathrm{Ar} / \mathrm{CO}$ flow. The region marked in gray corresponds to the stacking fault region in the Pt nanoparticle. Because of the finite voxel size of the reconstruction, the error bar on the vicinal surface assignment is a few degrees.

are relaxed outward, both to flatten the step. ${ }^{42-44}$ Apparently, the long-range strain fields of the step edges of the different vicinal NP facets interact and result in a compression of the near surface interatomic spacing by $-0.02 \%$ (see Figure $4 d$ ). In addition, the orientation of the internal twin boundary (gray area in Figure 5, see also the schematic view inside the bottom left circle) fits well to the (expected) [11 $\overline{1}]$ orientation.

Under $\mathrm{Ar} / \mathrm{CO}$ flow at $400 \mathrm{~K}, \mathrm{CO}$ adsorbs on the surface of the NP. ${ }^{45}$ Apparently, a slight shape change of the particle took place on the side opposite to the twin defect, with the appearance of $(22 \overline{1})$ and $(11 \overline{2})$ facets. Such a shape change is in line with previous studies reporting a high mobility of $\mathrm{Pt}$ atoms on vicinal $\mathrm{Pt}$ surfaces as well as $\mathrm{Pt}$ clusters under near ambient pressure $\mathrm{CO}$ exposure at room temperature. ${ }^{46-48}$ The high coverage adsorption of $\mathrm{CO}$ leads to a weakening of the bonds of the surface $\mathrm{Pt}$ atoms to their near surface neighbors, resulting in an outward relaxation of a few percent of the interlayer spacing as generally expected. ${ }^{49}$ On nanoparticles with well-defined edges and corners compressive effects were reported under oxygen exposure. ${ }^{40}$ The nanoparticle we have investigated exhibits a much rounder shape composed of vicinal surfaces, which exhibits a different relaxation behavior upon switching from $\mathrm{Ar}$ to $\mathrm{Ar} / \mathrm{CO}$ conditions. The near surface region of this analyzed NP is affected by an expansion of $+0.12 \%$, which is further transferred into the NP bulk (change of net compression from $-0.15 \%$ to $-0.02 \%$; see Figure $4 d$ ). The high strain sensitivity of CXDI visualizes thus nicely the influence of surface adsorbates on the near surface and bulk NP strain state, representing an additional degree of freedom not present for planar surfaces. $\mathrm{CO}$ adsorption induced shape changes of Pt NPs were recently observed by in situ transmission electron microscopy in a similar pressure and temperature regime, in line with our findings. ${ }^{50}$

\section{CONCLUSION}

We demonstrated that preselected, single Pt NPs can be investigated by coherent diffraction imaging at elevated temperatures under near atmospheric pressure gas flow conditions. The NP preselection overcomes the arbitrariness of previous experiments in searching a suitable NP and gives therefore access to much more systematic studies. Moreover, they allow a correlative investigation by complementary techniques such as atomic force and scanning electron or scanning tunneling microscopy. As we showed, defects, such as twin domain boundaries can be identified and artifacts in the CXDI data reconstruction can be avoided. Our experiments provide evidence that the interior strain state of STO supported Pt NPs is influenced by the presence of higher index facets at the NP surface. Upon exposure to mixed $\mathrm{Ar} /$ $\mathrm{CO}$ flow at near atmospheric pressures our data suggest a shape change of the Pt NP, simultaneously with the formation of facets, which are more stable under $\mathrm{CO}$ rich conditions. Accordingly, we observed a change in the NP strain state, with a $\mathrm{CO}$ adsorption induced outward relaxation of the atomic positions, being more pronounced in the near surface region of the NP. Our results demonstrate that the NP shape can influence the internal NP strain state, thus providing an additional "knob" for strain state tailoring, influencing reactivity. Future CXDI experiments will allow addressing the shape and the strain state of individual, preselected NPs under dynamical operando reaction conditions.

\section{ASSOCIATED CONTENT}

\section{S Supporting Information}

The Supporting Information is available free of charge on the ACS Publications website at DOI: 10.1021/acsanm.9b00764.

Figures S1-S15 (PDF)

Movie S1 (MP4)

Movie S2 (MP4)

Movie S3 (MP4)

Movie S4 (MP4)

\section{AUTHOR INFORMATION}

\section{Corresponding Authors}

*E-mail: ivan.vartaniants@desy.de.

*E-mail: andreas.stierle@desy.de.

ORCID

Young Yong Kim: 0000-0001-6102-9518

Simon Maier: 0000-0002-5647-8359

Dmitry Dzhigaev: 0000-0001-8398-9480

Sergey Lazarev: 0000-0002-7230-3559

Luca Gelisio: 0000-0001-7832-6201

Christoph Seitz: 0000-0002-8151-9933

Vedran Vonk: 0000-0001-9854-1101

Thomas F. Keller: 0000-0002-3770-6344

Ivan A. Vartanyants: 0000-0002-0340-8234

Andreas Stierle: 0000-0002-0303-6282

Notes

The authors declare no competing financial interest.

\section{ACKNOWLEDGMENTS}

Financial support from the European Union within the framework of the H2020 project "Nanoscience Foundries and Fine Analysis" (NFFA), grant no. 654360, and the use of 
the FIB dual beam instrument granted by BMBF under grant 5K13WC3 (PT-DESY) is gratefully acknowledged. Y.Y.K., D.D., S.L., L.G., and I.A.V. acknowledge the support by the Helmholtz Associations Initiative and Networking Fund and the Russian Science Foundation (Project No. 18-41-06001).

\section{REFERENCES}

(1) Ertl, G.; Knözinger, H.; Schüth, F.; Weitkamp, J. In Handbook of Heterogeneous Catalysis; Ertl, G., Knözinger, H., Schüth, F., Weitkamp, J., Eds.; Wiley-VCH: Weinheim, Germany, 2008.

(2) Lee, H.; Habas, S. E.; Kweskin, S.; Butcher, D.; Somorjai, G. A.; Yang, P. Morphological Control of Catalytically Active Platinum Nanocrystals. Angew. Chem. 2006, 118, 7988-7992.

(3) Tian, N.; Zhou, Z.-Y.; Sun, S.-G.; Ding, Y.; Wang, Z. L. Synthesis of Tetrahexahedral Platinum Nanocrystals with High-Index Facets and High Electro-Oxidation Activity. Science 2007, 316, 732-735.

(4) Lee, I.; Delbecq, F.; Morales, R.; Albiter, M. A.; Zaera, F. Tuning selectivity in catalysis by controlling particle shape. Nat. Mater. 2009, $8,132-138$.

(5) Jin, M.; Zhang, H.; Xie, Z.; Xia, Y. Palladium nanocrystals enclosed by $\{100\}$ and $\{111\}$ facets in controlled proportions and their catalytic activities for formic acid oxidation. Energy Environ. Sci. 2012, 5, 6352-6357.

(6) Bratlie, K. M.; Lee, H.; Komvopoulos, K.; Yang, P.; Somorjai, G. A. Platinum Nanoparticle Shape Effects on Benzene Hydrogenation Selectivity. Nano Lett. 2007, 7, 3097-3101.

(7) Tsung, C.-K.; Kuhn, J. N.; Huang, W.; Aliaga, C.; Hung, L.-I.; Somorjai, G. A.; Yang, P. Sub-10 nm Platinum Nanocrystals with Size and Shape Control: Catalytic Study for Ethylene and Pyrrole Hydrogenation. J. Am. Chem. Soc. 2009, 131, 5816-5822.

(8) Hejral, U.; Franz, D.; Volkov, S.; Francoual, S.; Strempfer, J.; Stierle, A. Identification of a Catalytically Highly Active Surface Phase for CO Oxidation over PtRh Nanoparticles under Operando Reaction Conditions. Phys. Rev. Lett. 2018, 120, 126101.

(9) Mavrikakis, M.; Hammer, B.; Norskov, J. K. Effect of Strain on the Reactivity of Metal Surfaces. Phys. Rev. Lett. 1998, 81, 2819-2822.

(10) Gsell, M.; Jakob, P.; Menzel, D. Effect of Substrate Strain on Adsorption. Science 1998, 280, 717-720.

(11) Jakob, P.; Gsell, M.; Menzel, D. Interactions of adsorbates with locally strained substrate lattices. J. Chem. Phys. 2001, 114, 1007510085 .

(12) Rhen, F. M. F.; McKeown, C. Enhanced Methanol Oxidation on Strained Pt Films. J. Phys. Chem. C 2017, 121, 2556-2562.

(13) Potapenko, D. V.; Gomes, G. T.; Osgood, R. M. Correlation of $\mathrm{H}$ Adsorption Energy and Nanoscale Elastic Surface Strain on Rutile $\mathrm{TiO}_{2}(110)$. J. Phys. Chem. C 2016, 120, 21373-21380.

(14) Khorshidi, A.; Violet, J.; Hashemi, J.; Peterson, A. A. How strain can break the scaling relations of catalysis. Nature Catalysis 2018, 1, 263-268.

(15) Pingel, T. N.; Jørgensen, M.; Grönbeck, H.; Olsson, E.; Yankovich, A. B. Influence of atomic site-specific strain on catalytic activity of supported nanoparticles. Nat. Commun. 2018, 9, 2722.

(16) Grunwaldt, J. D.; Wagner, J. B.; Dunin-Borkowski, R. E. Imaging Catalysts at Work: A Hierarchical Approach from the Macroto the Meso- and Nano-scale. ChemCatChem 2013, 5, 62-80.

(17) Schlögl, R. Heterogeneous Catalysis. Angew. Chem., Int. Ed. 2015, 54, 3465-3520.

(18) Li, X.; Chen, Q.; McCue, I.; Snyder, J.; Crozier, P.; Erlebacher, J.; Sieradzki, K. Dealloying of noble-metal alloy nanoparticles. Nano Lett. 2014, 14, 2569-2577.

(19) Fenger, R.; Fertitta, E.; Kirmse, H.; Thünemann, A. F.; Rademann, K. Size dependent catalysis with CTAB-stabilized gold nanoparticles. Phys. Chem. Chem. Phys. 2012, 14, 9343-9349.

(20) Ferrer, D.; Blom, D. A.; Allard, L. F.; Mejía, S.; Pérez-Tijerina, E.; José-Yacamán, M. Atomic structure of three-layer $\mathrm{Au} / \mathrm{Pd}$ nanoparticles revealed by aberration-corrected scanning transmission electron microscopy. J. Mater. Chem. 2008, 18, 2442-2446.
(21) Tao, F. F.; Crozier, P. A. Atomic-Scale Observations of Catalyst Structures under Reaction Conditions and during Catalysis. Chem. Rev. 2016, 116, 3487-3539.

(22) Robinson, I. K.; Vartanyants, I. A. Use of coherent X-ray diffraction to map strain fields in nanocrystals. Appl. Surf. Sci. 2001, $182,186-191$.

(23) Robinson, I.; Harder, R. Coherent X-ray diffraction imaging of strain at the nanoscale. Nat. Mater. 2009, 8, 291-298.

(24) Vartanyants, I. A.; Yefanov, O. M. In X-ray Diffraction: Modern Experimental Techniques; Seeck, O. H., Murphy, B., Eds.; Pan Stanford Publishing Pte. Ltd.: Singapour, 2015; Chapter 12, pp 341-384.

(25) Stierle, A.; Gustafson, J.; Lundgren, E. In Operando Research in Heterogeneous Catalysis; Frenken, J., Groot, I., Eds.; Springer International Publishing: Cham, 2017; pp 59-87.

(26) Ulvestad, A.; Sasikumar, K.; Kim, J. W.; Harder, R.; Maxey, E.; Clark, J. N.; Narayanan, B.; Deshmukh, S. A.; Ferrier, N.; Mulvaney, P.; Sankaranarayanan, S. K. R. S.; Shpyrko, O. G. In Situ 3D Imaging of Catalysis Induced Strain in Gold Nanoparticles. J. Phys. Chem. Lett. 2016, 7, 3008-3013.

(27) Ulvestad, A.; Tripathi, A.; Hruszkewycz, S. O.; Cha, W.; Wild, S. M.; Stephenson, G. B.; Fuoss, P. H. Coherent diffractive imaging of time-evolving samples with improved temporal resolution. Phys. Rev. B: Condens. Matter Mater. Phys. 2016, 93, 184105.

(28) Pfeifer, M. A.; Williams, G. J.; Vartanyants, I. A.; Harder, R.; Robinson, I. K. Three-dimensional mapping of a deformation field inside a nanocrystal. Nature 2006, 442, 63.

(29) Ulvestad, A.; Welland, M. J.; Collins, S. S. E.; Harder, R.; Maxey, E.; Wingert, J.; Singer, A.; Hy, S.; Mulvaney, P.; Zapol, P.; Shpyrko, O. G. Avalanching strain dynamics during the hydriding phase transformation in individual palladium nanoparticles. Nat. Commun. 2015, 6, 10092.

(30) Clark, J. N.; Ihli, J.; Schenk, A. S.; Kim, Y.-Y.; Kulak, A. N.; Campbell, J. M.; Nisbet, G.; Meldrum, F. C.; Robinson, I. K. Threedimensional imaging of dislocation propagation during crystal growth and dissolution. Nat. Mater. 2015, 14, 780.

(31) Dzhigaev, D; Shabalin, A; Stankevic, T; Lorenz, U; Kurta, R P; Seiboth, F; Wallentin, J; Singer, A; Lazarev, S; Yefanov, O M; Borgstrom, M; Strikhanov, M N; Samuelson, L; Falkenberg, G; Schroer, C G; Mikkelsen, A; Feidenhans'l, R; Vartanyants, I A Bragg coherent $\mathrm{x}$-ray diffractive imaging of a single indium phosphide nanowire. J. Opt. 2016, 18, 64007.

(32) Komanicky, V.; Iddir, H.; Chang, K.-C.; Menzel, A.; Karapetrov, G.; Hennessy, D.; Zapol, P.; You, H. Shape-dependent activity of platinum array catalyst. J. Am. Chem. Soc. 2009, 131, 57325733.

(33) Richard, M.-I.; Fernández, S.; Hofmann, J. P.; Gao, L.; Chahine, G. A.; Leake, S. J.; Djazouli, H.; De Bortoli, Y.; Petit, L.; Boesecke, P.; Labat, S.; Hensen, E. J. M.; Thomas, O.; Schülli, T. Reactor for nano-focused $\mathrm{x}$-ray diffraction and imaging under catalytic in situ conditions. Rev. Sci. Instrum. 2017, 88, 093902.

(34) Stierle, A.; Keller, T. F.; Noei, H.; Vonk, V.; Roehlsberger, R. DESY NanoLab. JLSRF 2016, 2, A76.

(35) Chahine, G. A.; Richard, M. I.; Homs-Regojo, R. A.; TranCaliste, T. N.; Carbone, D.; Jaques, V. L. R.; Grifone, R.; Boesecke, P.; Katzer, J.; Costina, I.; Djazouli, H.; Schroeder, T.; Schülli, T. U. Imaging of strain and lattice orientation by quick scanning X-ray microscopy combined with three-dimensional reciprocal space mapping. J. Appl. Crystallogr. 2014, 47, 762-769.

(36) Fienup, J. R. Phase retrieval algorithms: a comparison. Appl. Opt. 1982, 21, 2758-2769.

(37) Newton, M. C.; Leake, S. J.; Harder, R.; Robinson, I. K. Threedimensional imaging of strain in a single $\mathrm{ZnO}$ nanorod. Nat. Mater. 2010, 9, 120-124.

(38) Dupraz, M.; Beutier, G.; Rodney, D.; Mordehai, D.; Verdier, M. Signature of dislocations and stacking faults of face-centred cubic nanocrystals in coherent X-ray diffraction patterns: a numerical study. J. Appl. Crystallogr. 2015, 48, 621-644. 
(39) Ulvestad, A.; Clark, J. N.; Harder, R.; Robinson, I. K.; Shpyrko, O. G. 3D Imaging of Twin Domain Defects in Gold Nanoparticles. Nano Lett. 2015, 15, 4066-4070.

(40) Kim, D.; Chung, M.; Carnis, J.; Kim, S.; Yun, K.; Kang, J.; Cha, W.; Cherukara, M. J.; Maxey, E.; Harder, R.; Sasikumar, K.; K. R. S. Sankaranarayanan, S.; Zozulya, A.; Sprung, M.; Riu, D.; Kim, H. Active site localization of methane oxidation on Pt nanocrystals. Nat. Commun. 2018, 9, 3422.

(41) Westerberg, S.; Wang, C.; Somorjai, G. A. Heat of adsorption of $\mathrm{CO}$ on $\mathrm{Pt}\left(\begin{array}{lll}1 & 1 & 1\end{array}\right)$ obtained by sum frequency generation vibrational spectroscopy - A new technique to measure adsorption isotherms. Surf. Sci. 2005, 582, 137-144.

(42) Feibelman, P. J. Energetics of steps on Pt(111). Phys. Rev. B: Condens. Matter Mater. Phys. 1995, 52, 16845-16854.

(43) Prévot, G.; Steadman, P.; Ferrer, S. Determination of the elastic dipole at the atomic steps of $\mathrm{Pt}(977)$ from surface $\mathrm{x}$-ray diffraction. Phys. Rev. B: Condens. Matter Mater. Phys. 2003, 67, 245409.

(44) Vlad, A.; Stierle, A.; Westerström, R.; Blomberg, S.; Mikkelsen, A.; Lundgren, E. Oxygen interaction with the $\operatorname{Pd}(112)$ surface: From chemisorption to bulk oxide formation. Phys. Rev. B: Condens. Matter Mater. Phys. 2012, 86, 035407.

(45) Jensen, J. A.; Rider, K. B.; Salmeron, M.; Somorjai, G. A. High Pressure Adsorbate Structures Studied by Scanning Tunneling Microscopy: $\mathrm{CO}$ on $\mathrm{Pt}(111)$ in Equilibrium with the Gas Phase. Phys. Rev. Lett. 1998, 80, 1228-1231.

(46) Tao, F.; Dag, S.; Wang, L.-W.; Liu, Z.; Butcher, D. R.; Bluhm, H.; Salmeron, M.; Somorjai, G. A. Break-Up of Stepped Platinum Catalyst Surfaces by High CO Coverage. Science 2010, 327, 850-853.

(47) Balmes, O.; Prévot, G.; Torrelles, X.; Lundgren, E.; Ferrer, S. Generation of surface steps on $\mathrm{Pt}(977)$ induced by the catalytic oxidation of CO. J. Catal. 2014, 309, 33-37.

(48) Podda, N.; Corva, M.; Mohamed, F.; Feng, Z.; Dri, C.; Dvorák, F.; Matolin, V.; Comelli, G.; Peressi, M.; Vesselli, E. Experimental and Theoretical Investigation of the Restructuring Process Induced by $\mathrm{CO}$ at Near Ambient Pressure: Pt Nanoclusters on Graphene/Ir(111). ACS Nano 2017, 11, 1041-1053.

(49) Wang, J. X.; Robinson, I. K.; Ocko, B. M.; Adzic, R. R. Adsorbate-Geometry Specific Subsurface Relaxation in the CO/ Pt(111) System. J. Phys. Chem. B 2005, 109, 24-26.

(50) Avanesian, T.; Dai, S.; Kale, M. J.; Graham, G. W.; Pan, X.; Christopher, P. Quantitative and Atomic-Scale View of CO-Induced Pt Nanoparticle Surface Reconstruction at Saturation Coverage via DFT Calculations Coupled with in Situ TEM and IR. J. Am. Chem. Soc. 2017, 139, 4551-4558. 